\title{
INCIDENCIA DEL PARTO PREMATURO EN MUJERES EMBARAZADAS QUE HAN SUFRIDO VIOLENCIA EN URUGUAY
}

\section{INCIDENCE OF PRETERM BIRTH IN PREGNANT WOMEN WHO HAVE SUFFERED VIOLENCE IN URUGUAY}

\author{
Leticia Lorena Da Costa Leites Da Silva' \\ Anabel Sarduy Lugo'. \\ ${ }^{1}$ Universidad Estatal Península Santa Elena, Ecuador. \\ leticiadacosta23@gmail.com.
}

\section{RESUMEN}

Se evaluó el riesgo de parto prematuro en mujeres embarazadas uruguayas que hayan declarado sufrir violencia durante el año 2012. Se realizó un estudio descriptivo en Uruguay, durante el año 2012. La muestra estuvo constituida por 43.989 mujeres embarazadas registradas en el Sistema Informático Perinatal (S.I.P), que reportaron hechos violentos y presentaron los datos necesarios para el estudio. El análisis estadístico se realizó a través de Chi cuadrado, Varianza, Test de Student y Regresión binaria. Las mujeres que relataron situaciones de violencia sufridas en algún momento de la gestación, fueron 1,3\% del total de mujeres embarazadas. Casi el 19\% de los partos de las mujeres que sufrieron violencia fue de pre término; constituyéndose la violencia como el mayor riesgo social para la Prematurez Odds Ratio (OR): 1,34 (95\% IC 1,03-1,76). La asociación resultó fuerte e independiente de otras variables incluidas en el estudio. Los recién nacidos de estas mujeres presentaron bajo peso, deficiente perímetro cefálico e insuficiente longitud al nacer. La violencia en la mujer embarazada, como condición social, resultó ser el mayor riesgo social para el parto prematuro. Por las consecuencias adversas que genera la violencia, debería ser prioridad para los agentes de la salud.

Palabras clave: Violencia, mujer embarazada, parto prematuro.

\section{ABSTRACT}

Risk of preterm delivery in uruguayan pregnant women, who have declared being victims of violence during 2012. A descriptive study was conducted on Uruguay, during 2012 year. The sample was consisted of 43,989 pregnant women enrolled in Perinatal Information System, that report violence and present all the important information to the study. Statistical analysis performed using Chi square, Anova, Student test and binary regression. Women who reported violence experienced during gestation were $1.3 \%$ of all pregnant women. Almost $19 \%$ of births in women who suffered violence were preterm; constituting violence as the highest social risk Prematurity Odds Ratio (OR): 1.34 (95\% Cl 1.03 to 1.76). The association was strong and independent of variables included at study. Newborns of these women are underweight, insufficient cephalic perimeter and length at birth. Violence in pregnant women that live on this status proved to be the greatest social risk for preterm delivery. Because of the adverse consequences of violence, should be a priority for health workers.

Keywords: Violence, pregnant women, premature labor/delivery.

Recibido: 16 de noviembre de 2016

Aceptado: 3 de julio de 2017

Publicado: 31 de julio de 2017

\section{Introducción}

El objetivo de la investigación es evaluar el riesgo de parto prematuro en mujeres embarazadas uruguayas que hayan declarado sufrir violencia durante el año 2012, registradas en el S.I.P.
En el país no existen estudios que analicen la relación entre el parto prematuro y la violencia en las mujeres embarazadas, por ese motivo se decidió investigar sobre el tema.

Se tiene conocimiento que la violencia contra la mujer constituye tanto una violación a los derechos humanos de las mujeres como un 
grave problema de salud pública. Tiene un elevado costo social y económico, debido a las consecuencias negativas que produce, en todos los miembros de la familia y en la sociedad en su conjunto.

A nivel mundial la Organización Mundial de la Salud (OMS) estima que el $41 \%$ de las mujeres han sido víctimas de violencia y que durante el embarazo el $10 \%$ de las mujeres han sido golpeadas, siendo en el $96 \%$ de los casos el agresor el padre del hijo en gestación ${ }^{1}$. La violencia en las mujeres embarazadas se ha visto asociada al bajo peso al nacer (BPN) y a la prematurez. Esta relación tiene como posibles componentes etiológicos el trauma físico y el estrés. El trauma abdominal intenso puede causar resultados desfavorables en el embarazo. Varios estudios sugieren que el estrés psicológico materno está asociado con un aumento de riesgo de parto prematuro y de BPN . ${ }^{2}$ El estrés emocional puede activar el eje neuroendocrino, causando la liberación de catecolaminas, prostaglandinas y otros vasoconstrictores que conducen a la hipoxia fetal, a la restricción del crecimiento intrauterino y al trabajo de parto prematuro. ${ }^{3}$

Según el Ministerio del Interior, en el Uruguay los delitos de violencia están en aumento. Entre noviembre de 2012 y octubre de 2013, veintisiete mujeres fueron asesinadas por Violencia. ${ }^{4}$ El abuso físico es un tema difícil de denunciar. Por lo tanto, la verdadera asociación entre el abuso y el parto prematuro resulta compleja de estudiar.

El objetivo del presente trabajo, consiste en estudiar a través del Sistema Informático Perinatal (S.I.P), el riesgo de parto prematuro que presentan las mujeres embarazadas uruguayas, que hayan declarado sufrir violencia durante el año 2012.

\section{La violencia en la mujer embarazada como factor de riesgo para producir resultados adversos en la gestación: estrés psicosocial}

De acuerdo a Petersen, la relación entre la violencia durante el embarazo y los resultados adversos del mismo tienen dos componentes etiológicos: trauma físico y estrés. Respecto al trauma, se pudo observar que la intensidad del trauma abdominal es proporcional a los efectos desfavorables en la gestación. En cuanto al estrés materno, el autor destaca la evidencia es cada vez mayor sobre la asociación entre el estrés psicosocial y el parto prematuro. El estrés materno puede activar el eje neuroendocrino, causando la liberación de cortisol y catecolaminas, entre otros vasoconstrictores que aumentan las sustancias vasoactivas, que provocan la vasoconstricción en los vasos placentarios, lo cual conduce a la hipoxia fetal o restricción fetal del crecimiento, afectando negativamente los sistemas de órganos en crecimiento del feto, también predispone a la madre a tener el trabajo de parto prematuro. ${ }^{3}$

Zhu et al. ${ }^{5}$ sugieren que el estrés materno, tales como la ansiedad relacionada con el embarazo, depresión, o angustia, puede estar asociada con un aumento de riesgo de parto prematuro o de bajo peso al nacer.

\section{Relación entre violencia en la mujer embarazada y estrés materno, consecuencias en la gestación y en el neonato: La violencia en la mujer embarazada y los resultados perinatales en América Latina y el Caribe}

Han A y Sterwat D. ${ }^{6}$ realizaron una revisión de la literatura sobre los factores de riesgo y los resultados adversos de la violencia en la mujer en gestación.

Los resultados evidenciaron que los nacidos de las mujeres que sufrieron violencia durante el embarazo tenían 4 veces más probabilidad de tener B.P.N (IC 95\%, 1.3-12.3), 27,5\% tuvieron complicaciones neonatales como prematurez y 9,1\% presentaron mortalidad neonatal.

Se concluyó la alta prevalencia en América Latina y El Caribe (ALC) de violencia en la mujer embarazada, evidenciando malos resultados perinatales.

De acuerdo al estudio citado, la violencia en la mujer embarazada es factor de riesgo para el parto prematuro y este constituye la variable dependiente más importante para nuestra investigación.

\section{Métodos}

Se realizó un estudio descriptivo en los 19 departamentos del Uruguay, durante el período de un año.

El universo lo constituyeron un total de 55.780 mujeres embarazadas registradas en el S.I.P que reportaron hechos violentos durante el año 2012. La muestra estuvo constituida por 43.989 mujeres embarazadas registradas en el mismo sistema, durante el mismo período, que reportaron hechos violentos y presentaban todos los datos necesarios para realizar el estudio. 
Para recopilar la información necesaria durante el estudio, se utilizó el programa SPSS versión 18.0 para Windows.

Las principales variables de estudio fueron Violencia y Prematurez. Las variables secundarias fueron: Consumo de tabaco, alcohol y drogas, embarazo adolescente, escaso nivel de escolaridad materna, B.P.N, longitud al nacer deficiente, perímetro cefálico insuficiente al nacer, antecedentes de aborto, mortinato, escaso controles durante el embarazo, consideró antecedentes de hipertensión, preeclampsia o eclampsia, presencia de hipertensión durante el embarazo, anemia durante el embarazo, infecciones de transmisión sexual durante el embarazo, soltera.

Se consideró Violencia, cuando en el S.I.P estaba registrado que la mujer embarazada durante el control prenatal declaró haber vivido situaciones de violencia.

Se consideró Prematurez, cuando el nacimiento tuvo lugar antes de completadas las 37 semanas de gestación. ${ }^{7}$

Las variables de control fueron:

-Consumo de tabaco, alcohol y drogas. Las mismas fueron definidas dentro del grupo:"abuso de sustancias".

Se consideró consumo de tabaco, cuando la mujer declaró haber fumado en el primer $y / 0$ segundo $y / o$ tercer trimestre del embarazo.

Se consideró consumo de alcohol, cuando la mujer declaró haber bebido alcohol en el primer y/o segundo y/o tercer trimestre del embarazo.

Se consideró consumo de drogas, cuando la mujer declaró haber consumido drogas en el primer $y / 0$ segundo $y / 0$ tercer trimestre del embarazo.

-Se consideró mujeres embarazadas adolescentes, cuando la gestación se inició entre los 10 y los 19 años. ${ }^{8}$

-Se consideró bajo nivel de escolaridad materna cuando la mujer sólo presentó estudios de nivel de primaria.

-Bajo peso al nacer. Se consideró cuando el recién nacido pesó menos de 2500 grs. $^{9}$

-Longitud al nacer deficiente. Se consideró cuando el recién nacido midió menos de $45 \mathrm{~cm}$ de longitud. ${ }^{9}$
-Perímetro cefálico insuficiente al nacer. Se consideró, cuando el perímetro cefálico del recién nacido resultó menos a 33 $\mathrm{cm} .{ }^{9}$

-Se consideró antecedentes de aborto, cuando la mujer embarazada declaró haber tenido uno o más abortos; definiéndose aborto como la pérdida o interrupción de un embarazo clínico, que tiene lugar antes de completar las 20 semanas de edad de gestación. ${ }^{10}$

-Se consideró mortinato, cuando la mujer embarazada declaró la expulsión o extracción del feto, a partir de la semanas 20 de edad gestacional. ${ }^{10}$

-Se consideró escasos controles durante el embarazo, cuando el número de los controles registrados en el carnet obstétrico de la mujer embarazada, fueran 5 o menos.

-Se consideró antecedentes de hipertensión, preeclampsia o eclampsia, cuando estaban registrados en la historia clínica.

-Se consideró presencia de hipertensión durante el embarazo, cuando en el carnet obstétrico se registró cifras de presión arterial $>0=140 / 90 \mathrm{mmHg}$ o estaba registrado que la mujer presentaba preeclampsia 0 eclampsia. ${ }^{11}$

-Se consideró anemia durante el embarazo, cuando las cifras de hemoglobina $(\mathrm{Hb})$ fueron menores a $11 \mathrm{~g} / \mathrm{dl}$ durante el embarazo. ${ }^{11}$

-Se consideró presencia de infecciones de transmisión sexual durante el embarazo, cuando en el carnet obstétrico se registró sífilis (treponémica o no treponémica) en tratamiento o no. ${ }^{11}$

-Se consideró soltera, cuando la mujer embarazada declaró vivir sola o no estaba en unión estable.

\section{Análisis estadístico}

Para el análisis de las variables cuantitativas se estimaron medidas descriptivas de resumen como media, mediana y desvío estándar (DE). Las variables cualitativas se expresaron en frecuencia absoluta y porcentajes.

La asociación de las variables cualitativas se realizó a través de la prueba de hipótesis de Chi-cuadrado de Pearson (chi ${ }^{2}$. Se aplicará el análisis de varianza y test de t de Student para las variables cuantitativas.

Para conocer el riesgo de prematurez se realizó un modelo de regresión logística binaria. La 
chance fue expresada como Odds Ratios (OR) con un intervalo del $95 \%$ de confianza. Todos los test se considerarán estadísticamente significativos con un nivel $(\alpha)$ de $0.05(p<0.05)$.

Para el procesamiento de los datos se utilizará el programa SPSS versión 18.0 para Windows.

\section{Aspectos éticos}

Los datos para la investigación fueron extraídos del S.I.P, a través de una autorización del Ministerio de Salud para nuestro estudio. La información que el organismo nos brindó sobre las mujeres embarazadas fue anónima.

\section{Resultados}

En el año 2012 de las 43.989 mujeres seleccionadas para el estudio, sólo 535 (1,3\%) relataron situaciones de violencia sufridas en algún momento de la gestación; 247 mujeres en el primer trimestre $(0,6 \%), 154$ en el segundo $(0,4 \%)$ y 138 en el tercero $(0,3 \%)$.
Casi el $19 \%$ de los partos de las mujeres que sufrieron violencia fue de pre término; constituyéndose la violencia como el mayor riesgo social para la Prematurez Odds Ratio (OR): 1,34 (95\% IC 1,03-1,76). La asociación resultó fuerte e independiente de algunas variables como ser adolescente, soltera, solo con educación primaria y padecer sífilis.

En cuanto a las características de las mujeres embarazadas, se pudo observar que el $28,5 \%$ de las mujeres que sufrieron violencia eran adolescentes $(p<0,001)$, el $48 \%$ sólo alcanzó a terminar primaria $(p=0,01)$, el $37 \%$ eran solteras $(p<0,001)$ y el $41,3 \%$ tuvo un embarazo mal controlado $(p=0,01)$. En lo que respecta a la salud el $48 \%$ tenía antecedentes de preeclampsia $(p<0,001)$ y el $7,3 \%$ sífilis $(p=0,001)$. Un alto porcentaje de estas mujeres declararon abuso de sustancias tóxicas: el $56,3 \%$ eran fumadoras $(p<0,001)$, el $30,8 \%$ consumía alcohol $(p<0,001)$ y el $30,2 \%$ drogas $\mathrm{p}=0,001)$ Tabla 1.

Tabla 1 Características de las mujeres según condición de violencia durante el embarazo

\begin{tabular}{|l|l|l|l|}
\hline Variables & Violencia & No violencia & Valor $\mathbf{p}$ \\
\hline Ser adolescente & $51(28,5 \%)$ & $7521(18,1 \%)$ & $<0,001$ \\
\hline Solo educación primaria & $83(48 \%)$ & $8829(21,4 \%)$ & 0,01 \\
\hline Soltera & $64(37,0 \%)$ & $7266(17,9 \%)$ & $<0,001$ \\
\hline$<6$ controles en embarazo & $74(41,3 \%)$ & $9592(23,0 \%)$ & 0,01 \\
\hline $\begin{array}{l}\text { Antecedentes } \\
\text { preeclampsia }\end{array}$ & $14(7,9 \%)$ & $1206(2,9 \%)$ & $<0,001$ \\
\hline Sífilis & $13(7,3 \%)$ & $725(1,7 \%)$ & 0,001 \\
\hline Fumadora & $98(56,3 \%)$ & $7844(18,9 \%)$ & $<0,001$ \\
\hline Alcohol & $53(30,8 \%)$ & $290(0,7 \%)$ & $<0,001$ \\
\hline Drogas & $52(30,2 \%)$ & $340(0,8 \%)$ & 0,001 \\
\hline
\end{tabular}

Un alto porcentaje de los partos de las mujeres que sufrieron violencia fue de pre término $(18,8 \%)$ y los recién nacidos resultaron con bajo peso $(12,3 \%)$; deficiente perímetro cefálico $(19,5 \%)$ e insuficiente longitud al nacer (10,3\%) al compararlos con los neonatos de las mujeres que no padecieron maltrato $8,8 \%, 6,7 \%, 11,9 \%$ y $5,3 \%$ respectivamente) siendo los resultados estadísticamente significativos $(p<0,001$, $\mathrm{p}=0,003, \mathrm{p}=0,002$ y $\mathrm{p}=0,006)$ Tabla 2 .

Tabla 2 Características de los niños/as nacidos de las mujeres que sufrieron violencia durante el embarazo

\begin{tabular}{|l|l|l|l|}
\hline Variables & Violencia & No violencia & Valor $\mathbf{p}$ \\
\hline Bajo peso al nacer & $22(12,3 \%)$ & $2798(6,7 \%)$ & 0,003 \\
\hline PC deficiente & $33(19,5 \%)$ & $4674(11,9 \%)$ & 0,002 \\
\hline Longitud al nacer deficiente & $18(10,3 \%)$ & $2283(5,6 \%)$ & 0,006 \\
\hline Prematurez & $33(18,8 \%)$ & $3679(8,8 \%)$ & $p<0,001$ \\
\hline
\end{tabular}


El mayor riesgo social para el parto prematuro resultó ser la violencia. Como se vió anteriormente el $18,8 \%$ de las mujeres que sufrieron maltrato tuvieron hijos prematuros en relación al $8,8 \%$ de las mujeres que no lo declararon $(\mathrm{p}<0,0001)$ Tabla 2.

Los mayores niveles de Prematurez se observaron entre los niños nacidos de mujeres con eclampsia $(36,5 \%) \quad(p<0,001)$, preeclampsia $\quad(33,2 \%) \quad(p<0,001), \quad$ con antecedentes de hipertensión (18,3\%) $\quad(p<$ $0,001)$, embarazos mal controlados $(18 \%)(p<$ $0,001)$, consumo de drogas $(17,4 \%)(p<0,001)$ $y$ alcohol $(16,7 \%) \quad(p<0,001)$. Asimismo, el $12,7 \%$ de las mujeres con sífilis ( $p=0,003$ ), el $10,5 \%$ con anemia $(p=0,003)$ y el $11,3 \%$ de las adolescentes $(p<0,001)$ tuvieron hijos prematuros Tabla 3.

Tabla 3: Porcentaje de partos prematuros según condición de la mujer

\begin{tabular}{|l|l|l|l|}
\hline Variables & Parto prematuro & Parto a término & Valor $\mathbf{p}$ \\
\hline Eclampsia & $23(36,5 \%)$ & $40(63,5 \%)$ & $\mathrm{p}<0,001$ \\
\hline Preeclampsia & $380(33,2 \%)$ & $764(66,8 \%)$ & $\mathrm{p}<0,001$ \\
\hline Antecedentes de hipertensión & $157(18,3 \%)$ & $703(81,7 \%)$ & $\mathrm{p}<0,001$ \\
\hline Embarazos mal controlados & $1921(18,0 \%)$ & $8772(82,0 \%)$ & $\mathrm{p}<0,001$ \\
\hline Consumo de drogas & $76(17,4 \%)$ & $362(82,6 \%)$ & $\mathrm{p}<0,001$ \\
\hline Consumo de alcohol & $65(16,7 \%)$ & $325(83,3 \%)$ & $\mathrm{p}<0,001$ \\
\hline Sífilis & $102(12,7 \%)$ & $198(87,3 \%)$ & $\mathrm{p}=0,003$ \\
\hline Anemia & $326(10,5 \%)$ & $2775(89,5 \%)$ & $\mathrm{p}=0,003$ \\
\hline Ser Adolescente & $922(11,3 \%)$ & $7202(88,7 \%)$ & $\mathrm{p}<0,001$ \\
\hline
\end{tabular}

En la Tabla 4 se puede observar los Odds Ratios ajustados para la violencia según análisis de regresión logística binaria. Las mujeres que sufrieron violencia en el embarazo mostraron un tercio más de chances de prematurez OR: 1,34 (IC 95\% 1,03-1,76) después de ajustar por adolescencia OR: 1,16 (IC 95\% 1,06-1,27), sólo educación primaria OR: 1,15 (IC 95\% 1,06-1,25), sífilis OR: 1,11 (IC $95 \% 1,012-1,21$ ) y ser soltera OR: 1,32 (IC $95 \%$ $1,05-1,67)$.

Tabla 4 Riesgo de parto prematuro en mujeres que sufren violencia

\begin{tabular}{|l|c|c|c|}
\hline Variables & B & Sig & OR (IC 95\%) \\
\hline Sufrir violencia & 0,295 & 0,14 & $1,34(1,03-1,76)$ \\
\hline Adolescente & 0,146 & 0,04 & $1,16(1,06-1,27)$ \\
\hline Solo primaria & 0,143 & 0,04 & $1,15(1,06-1,25)$ \\
\hline Sífilis & 0,281 & 0,12 & $1,11(1,012-0,21)$ \\
\hline Soltera & 0,102 & 0,05 & $1,32(1,05-1,67)$ \\
\hline
\end{tabular}

\section{Conclusión}

En el presente trabajo las mujeres que sufrieron violencia, presentaron un altísimo riesgo social ya que eran muy jóvenes, solteras, con bajo nivel educativo, escasos controles prenatales, con alto consumo de tabaco, alcohol y drogas. Además evidenciaron graves problemas de salud como sífilis y preeclampsia.

Según la evidencia científica. ${ }^{(2,3,5)}$ las consecuencias de eventos estresantes, como la violencia, repercute tanto en la duración del embarazo como en el tamaño corporal del recién nacido. En el presente trabajo el 18,8\% de los niños fueron prematuros y el $12,3 \%$ nació de bajo peso. Al igual que en la investigación realizada por Lederman ${ }^{5}$, los niños de madres que sufrieron violencia también tuvieron perímetro cefálico insuficiente. La circunferencia de la cabeza al nacer y en el primer año de vida, es considerada un 
importante predictor del éxito de los niños en la escuela.

El nacimiento prematuro constituye la causa principal de muerte de los recién nacidos. A nivel mundial, según el informe de la OMS el rango de nacimientos prematuros es de 5 al $18 \% .^{6,14-15}$ En el presente trabajo, las mujeres que sufrieron violencia, evidenciaron cifras más altas que el rango máximo a nivel mundial. La asociación entre violencia y el parto prematuro resultó fuerte e independiente de las características sociodemográficas (ser adolescente, soltera y sólo con educación primaria) y de las condiciones de salud como sífilis. Sin embargo, el riesgo de Prematurez provocado por la violencia resultó más bajo que el observado en otras investigaciones. ${ }^{6}$ Ello podría deberse a que en nuestro estudio más de la mitad de las mujeres que sufrieron violencia fumaban, casi un tercio consumían alcohol y drogas y casi un 8\% tenían antecedentes de preeclampsia, situaciones de altísimo riesgo para el parto prematuro. ${ }^{2-3,5-6}$

El abuso de sustancias, no sólo produce condiciones adversas perinatales, sino también repercute en el desarrollo del niño/a durante las etapas posteriores de la vida, además de afectar negativamente la salud de la mujer. En cuanto al consumo de tabaco, Kitsantas et al. ${ }^{16}$ encontraron que el $12 \%$ de los neonatos nacidos de mujeres fumadoras fueron prematuros, un porcentaje similar al de nuestra investigación (11\%). Respecto al consumo de alcohol, O'Leary $\mathrm{CM}$ et al. ${ }^{17}$ hallaron que el porcentaje de nacimientos prematuros fue más alto en niños expuestos durante el primer trimestre del embarazo, en comparación a los expuestos en el tercer trimestre $(13,6 \%$ vs $9,5 \%$ ). En nuestra investigación, encontramos que el $16,7 \%$ de las mujeres que consumieron alcohol durante el embarazo tuvieron hijos prematuros. Huestis y Choo. ${ }^{12}$ hallaron que 20 $\%$ de los niños expuestos a las drogas durante el embarazo fueron prematuros, resultado similar al observado en el presente trabajo $(17,4 \%)$.

En la investigación las mujeres que padecían violencia eran con frecuencia adolescentes, lo cual puede significar un riesgo potencial para la prematurez. El $11,6 \%$ de las adolescentes tuvieron parto prematuro, resultado similar a los estudios de otros autores. ${ }^{14}$ Shrim $A$ et al. ${ }^{18}$ demostraron que las madres adolescentes tenían cuatro veces más de probabilidad de tener parto prematuro (OR: 4,5 IC 95\% 2.5-8.1) y Zhu P et al. ${ }^{5}$ tres veces (RR: 2,74 IC $95 \%$ 1,57-4,78).
De acuerdo al presente trabajo, sólo el 1,3\% de las mujeres embarazadas registradas en el SIP en el año 2012, declararon vivir situaciones de violencia. Este porcentaje resultó extremadamente bajo al compararlo con las cifras mundiales, regionales y nacionales. ${ }^{1-2}$. En nuestro país los delitos por violencia están en aumento ${ }^{4}$. Algunas de las razones para el subregistro podrían ser por un lado, que las mujeres son menos propensas a reportar abuso por temor a las consecuencias y por otro que, los actores de la salud omiten detectar e informar de estas situaciones ya que no las consideran como prioridad en la consulta obstétrica.

El primer paso para afrontar el reto de la disminución y prevención de la violencia de género, es detectarla en forma oportuna $y$ adecuada. Los trabajadores de la salud, especialmente los que están en contacto con la mujer embarazada, desempeñan un papel crucial. El médico puede ser la única persona que la mujer logre acudir fuera de su familia; por lo que tienen una oportunidad única y la responsabilidad de intervenir. ${ }^{19}$ El embarazo es el momento ideal para detectar la violencia de género; ya que en el $25 \%$ de los casos, los malos tratos comienzan en el primer trimestre de la gestación. ${ }^{11}$ En Venezuela se ha revelado que los médicos de familia sólo realizan cribado de violencia al $10 \%$ de las mujeres, los ginecólogos el $17 \%$ y los internistas el $6 \% .^{20}$ Observando estas cifras y los resultados de nuestro trabajo reconocemos como falencia en nuestro Sistema de Salud, la falta de detección, hecho indispensable para la prevención de la violencia y para la protección de las mujeres.

Otras medidas para la prevención de la violencia de género, podrían ser la capacitación para la detección, atención, derivación y denuncia; lo que incluye el protocolo de abordaje de situaciones de violencia doméstica hacia la mujer del Ministerio de Salud Pública (M.S.P) y una agenda de recursos para tales situaciones. ${ }^{19}$ También se podrían realizar, programas de sensibilización y formación en violencia a los prestadores de servicios de salud, efectuar diversas campañas de información y sensibilización sobre los derechos de la mujer a una vida libre de violencia, realizar investigaciones y recopilación de estadísticas pertinentes sobre las causas, consecuencias y frecuencia de la violencia contra la mujer, con el fin de proponer y aplicar los cambios que sean necesarios. ${ }^{(19)}$

La normativa nacional actual es acorde a los compromisos internacionales, por lo tanto, debemos seguir trabajando en cooperación, realizando investigaciones que nos permitan 
enriquecernos mutuamente para abordar de mejor manera esta problemática. ${ }^{(21,22)}$

De acuerdo a nuestros hallazgos científicos, la violencia en la mujer embarazada, como condición social, resultó ser el mayor riesgo para el parto prematuro. La asociación resultó fuerte e independiente de algunas variables sociodemográficas y de salud. Por las consecuencias negativas en la salud de la mujer, de sus hijos, y del detrimento que genera en el capital social, la prevención, la detección y el abordaje de la violencia deberían ser prioritarios para los agentes comunitarios y de la salud.

\section{Agradecimientos}

A Dios en primer lugar, a mi familia y a la Dra. María Isabel Bove por motivarme en el área de la investigación.

\section{Referencias}

1. Organización Mundial de la Salud. Estudio multipaís de la OMS sobre salud de la mujer y violencia doméstica. Departamento Género, Mujer y Salud. Ginebra: OMS; 2005.

2. www.who.int/gender/violence/who multicou ntry study/summary report/summaryreport Spanishlow.pdf

3. Rodrigues $T$, Rocha $L$, Barros $H$, et al. Physical abuse during pregnancy and preterm delivery. Am J Obstet Gynecol 2008; 198:171 -176. Disponible en: www.ncbi.nlm.nih.gov/pubmed/17905171

4. Fríes L, Hurtado V. Estudio de la información sobre la violencia contra la mujer en América Latina y el Caribe. CEPAL.ONU. División de asuntos de género. Santiago de Chile, 2010. Disponible en: www.cepal.org/es/publicaciones/5826estudio-la-informacion-la-violencia-la-mujeramerica-latina-caribe

5. Ministerio del Interior. Observatorio nacional de violencia y criminalidad del Uruguay. Montevideo, 2013. Disponible en: www.minterior.gub.uy/observatorio/images/ stories/anual 2013.pdf

6. Zhu P, Tao F, Hao J, et al. Prenatal life events stress: implications for preterm birth and infant birthweight. Am J Obstet Gynecol. 2010; 203:34-38
7. Han A y Sterwat D. La violencia en la mujer embarazada y los resultados perinatales en América Latina y el Caribe. Am J Obstet Gynecol. 2014; 124: 6-11.

8. Ministerio de Salud Pública. Programa Nacional de la Salud y de la niñez. Área ciclos de vida, 2011.

9. Organización Panamericana de la Salud. Salud del Adolescente. OPS/OMS Washington: OMS; 1999. Disponible en: www.paho.org/hq/index.php?option=com d ocman\&task=doc view\&gid $=16931 \&$ Itemid

10. Organización Mundial de la Salud. Patrones de crecimiento de la OMS. Washington: OMS; $2007 . \quad$ Disponible en: www.who.int/childgrowth/standards/es/

11. Organización Mundial de la Salud. Glosario de terminología en técnicas de reproducción asistida. Ginebra: OMS; 2009. Disponible en:

www.who.int/reproductivehealth/publication s/infertility/art terminology2/es/

12. Ministerio de Salud Pública. Normas de Atención a la Mujer Embarazada. Montevideo; 2007. Disponible en: www.sguruguay.org/documentos/msp-gssrcapitulo-normas-atencion-mujerembarazada.pdf

13. Narkowicz S, Plotka J, Polkowska Z, Biziuk M, Namiesnik J. Prenatal exposure to substance of abuse: a worldwide problem. Poland; 2013. Disponible en: www.ncbi.nlm.nih.gov/pubmed/23454110

14. Hayatbakhsh MR, Flenady VJ, Gibbons KS, Kingsbury AM, Hurrion E, Mamun AA, Najman JM.Birthoutcomes associated with cannabis use before and during pregnancy Am J Obstet Gynecol. 2012; 71 (2):215-19. Disponible en: www.ncbi.nlm.nih.gov/pubmed/22258135

15.Kinney, M.V., Howson, C.P,McDougall, L., y Lawn, J.E. Informe de acción global sobre nacimientos prematuros. Nacidos demasiado pronto, OMS; 2012. Disponible en:

www.who.int/pmnch/media/news/2012/born toosoon execsum es.pdf

16. Ministerio de Salud Pública. Programa Nacional de la Salud y la niñez. Área ciclos de vida. Montevideo; 2011

17. Kitsantas $\mathrm{P}$, Christopher KE. Smoking and respiratory conditions in pregnancy: associations with adverse pregnancy outcomes. Southern Medical Journal. 2013; 
106(5): 310-15. Disponible en: www.ncbi.nlm.nih.gov/pubmed/23644639

18. O'Leary CM, Nassar N, Kurinczuk JJ, Bower C. The effect of maternal alcohol consumption on fetal growth and preterm birth. RCOG. 2009; 390-400. Disponible en: www.ncbi.nlm.nih.gov/pubmed/19187371

19.Shrim A, Ates $S$, Mallozzi A, Brown $R$, Ponette V, Almog B, et al. Is young maternal age really a risk factor for adverse pregnancy outcome in a canadian tertiary referral hospital? J Pediatr Adolesc Gynecol.2011. Disponible en: www.ncbi.nlm.nih.gov/pubmed/21620742

20. Ministerio de Salud Pública. Abordaje de situaciones de violencia domestica hacia la mujer. Montevideo, 2006. Disponible en: www.dem.fmed.edu.uy/materno/Ginecologi aObstetricia/Asistencia\%20a\%20\%20mujer es\%20en\%20situacion\%20de\%20violencia \%20domestica.pdf
21. Sociedad de obstetricia y ginecología de Venezuela. Violencia basada en género desde la perspectiva médica. Revista de Obstetricia y Ginecología de Venezuela. Caracas; $2011 . \quad$ Disponible en: www.scielo.org.ve/scielo.php?script=sci art text\&pid=S0048-77322011000200003

22.Poder Legislativo. Ley № 17.514 Violencia Domestica. Boletín oficial del Estado № 26045. Publicada D.O. 9 julio 2002.Uruguay. Disponible en: www.cepal.org/oig/doc/Uru2002Ley1754Vio lencia\%20Domestica.pdf

23. Suprema Corte de Justicia del Uruguay, bancada femenina del Parlamento Nacional del Uruguay y M.I.D.E.S. Violencia domestica ¿Sanción o impunidad? Seminario de consulta regional. Montevideo, $2005 . \quad$ Disponible en: www.adasu.org/prod/1/133/Diag.Respuesta .del.Estado.a.la.violencia..pdf 